\title{
Tratamento de Superfície de Tubos de Poliamida 11 com Chama para Deposição de Revestimento Hidrof́líco Biomédico
}

\author{
Waldemar F. M. Martins, Débora M. Fúcio, Frederico S. Mattoso, William S. Wiggers \\ Nano Endoluminal S.A.
}

Gean V. Salmória CIMJECT, UFSC

\begin{abstract}
Resumo: O processo para obtenção de uma camada polimérica hidrofílica sobre um componente polimérico pode ser basicamente dividido em: tratamento de superfície do substrato, aplicação e cura do revestimento. O tratamento de superfície se faz necessário em função da grande diferença de polaridade comumente apresentada entre os materiais que compõem este conjunto e da baixa energia livre de superfície inerente aos principais materiais utilizados como substrato, fatores estes que dificultam a adesão entre eles. Neste trabalho foi avaliada a influência da técnica de tratamento de superfície através de exposição à chama sobre a tentativa de aumento da energia livre de superfície de tubos de poliamida 11. Amostras foram submetidas ao tratamento durante 5, 10, 20, 30 e 60 segundos, e posteriormente caracterizadas por goniometria, microscopia eletrônica de varredura, espectroscopia de infravermelho e resistência ao risco da camada depositada. Amostras não tratadas foram analisadas como parâmetro de controle das modificações. Os resultados mostraram um aumento da molhabilidade e rugosidade da superfície, além de uma melhoria na adesão da camada hidrofílica após o tratamento.
\end{abstract}

Palavras-chave: Poliamida 11, tratamento de superfície, revestimento hidrofílico.

\section{Flame Surface Treatment of Polyamide 11 Tubes for the Deposition of Biomedical Hydrophilic Coatings}

Abstract: The process of obtaining a hydrophilic polymer coating on a polymeric component or product can be basically divided into: the substrate surface treatment, the coating application and the cross-linking. The substrate surface treatment is crucial due to the possible large difference in polarity between the polymers and the substrate materials, which commonly have low free surface energy and may severely hamper adhesion. This study evaluated the influence of flame surface treatment for the attempt of increasing the free energy surface of polyamide 11 tubes. Samples were submitted to treatment for 5, 10, 20, 30 and 60 seconds, and subsequently characterized by goniometry, scanning electron microscopy, infrared spectroscopy and scratch resistance of the deposited layer. Untreated samples were analyzed as a control parameter of the changes. The results showed an increase in wettability and surface roughness, and also an improvement in the adhesion of the hydrophilic coating after treatment.

Keywords: Polyamide 11, surface treatment, hydrophilic coating.

\section{Introdução}

A incorporação de procedimentos menos invasivos à prática cirúrgica de forma geral tem proporcionado a médicos e principalmente a pacientes, muitas facilidades e benefícios frente às tradicionais técnicas equivalentes. Por meio de pequenas incisões localizadas e de equipamentos específicos, o manuseio cirúrgico fica restrito apenas à área enferma, preservando assim as estruturas periféricas. Dentre as inúmeras vantagens apresentadas por estas técnicas destaca-se a redução da necessidade de reposição de sangue, a diminuição do tempo de internação, da probabilidade de infecções e das dores pós-operatórias ${ }^{[1]}$. Por outro lado, estas técnicas também apresentam algumas limitações da sua plena utilização. Tratando-se dos procedimentos endovasculares, por exemplo, um dos maiores problemas enfrentados são os traumas e lesões causados às paredes dos vasos sanguíneos devido à fricção do dispositivo durante sua introdução ${ }^{[1]}$.

Alguns recursos tecnológicos na área dos materiais têm ajudado na diminuição desses danos, como por exemplo, por meio da aplicação de uma camada polimérica hidrofílica sobre a superfície do dispositivo. Materiais hidrofílicos são polares, e apresentam grande afinidade com líquidos de mesmo caráter, como o sangue, o que torna a interface entre estes altamente deslizantes ${ }^{[2]}$. Além disso, os polímeros hidrofílicos apresentam resultados positivos em ensaios como citotoxicidade, toxicidade sistêmica, reatividade intracutânea aguda, entre outros, comprovando que suprem também exigências associadas à biocompatibilidade ${ }^{[1]}$.

De forma geral, a obtenção de um revestimento polimérico hidrofílico sobre outro componente polimérico pode ser dividida em três etapas: o tratamento de superfície do substrato, a deposição do revestimento e a cura do polímero depositado. Dentre estas etapas, o tratamento de superfície se faz necessário, em função, sobretudo, da diferença de polaridade comumente apresentada entre estes materiais, e da baixa energia livre de superfície dos principais polímeros utilizados como substrato.

O tratamento de superfície de polímeros pode ser realizado por vários métodos desde processos químicos por via úmida, através da ação de substâncias oxidantes, até processos a seco, como a oxidação eletroquímica ou anôdica ${ }^{[3]}$, tratamento térmico ${ }^{[4]}$, por chama $^{[5]}$, polimerização a plasma ${ }^{[6,7]}$ e a laser ${ }^{[8,9]}$. Tal tratamento provoca modificações químicas na superfície do material, induzindo a formação de grupos funcionais polares capazes de aumentar a interação entre os polímeros do substrato e do revestimento por meio de ligações secundárias ${ }^{[3]}$, melhorando a molhabilidade do substrato $^{[10]}$ e consequentemente a aderência da camada polimérica hidrofílica depositada ${ }^{[11]}$. A molhabilidade, que é a habilidade de um líquido de aderir em um sólido espalhando-se ao longo de sua superfície em diferentes graus, se relaciona diretamente com o grau de hidrofilicidade do material ${ }^{[12]}$. Esses tratamentos promovem também um aumento da rugosidade superficial, e 
consequentemente da área de contato, aumentando assim a energia livre de superfície ${ }^{[12]}$.

O presente estudo avalia o tratamento de superfície de tubos de poliamida 11 por exposição à chama. Foi analisado o aumento da energia livre da superfície dos tubos após o tratamento, bem como a contribuição das modificações de relevo sobre essas alterações de energia, objetivando favorecer a adesão de um revestimento polimérico hidrofílico. Dentre os vários tipos de tratamentos de superfície comumente empregados no processamento de polímeros, o tratamento por chama foi selecionado para este estudo em decorrência da sua relativa facilidade na montagem do aparato, do baixo custo dos equipamentos e por ser uma técnica de rápida execução. Amostras de tubos de poliamida 11 foram tratadas por chama durante $5,10,20,30$ e 60 segundos. Amostras tratadas e não tratadas (parâmetro de controle) foram recobertas com uma fina camada de polímero hidrofílico, que posteriormente sofreu o processo de cura (processo não avaliado neste trabalho). As técnicas empregadas para caracterização foram: goniometria, microscopia eletrônica de varredura, espectroscopia de infravermelho com transformada de Fourier por reflectância (FTIR) e teste de resistência ao risco.

\section{Experimental}

\section{Materiais}

Nesse estudo, o material utilizado como substrato para o tratamento de superfície foi a poliamida 11 (PA11) na forma de tubos com as dimensões de $1130 \mathrm{~mm}$ de comprimento, 0,6 $\mathrm{mm}$ de espessura de parede e 3,8 mm de diâmetro externo. Estes tubos são fornecidos pela empresa AP Extrusion Incorporated (EUA) e classificados como adequados para o emprego na fabricação de dispositivos médicos de acordo com o United States Food and Drug Administration (FDA). Primeiramente, os tubos foram segmentados em corpos de prova com comprimento de $40 \mathrm{~mm}$ e posteriormente limpos com álcool etílico de concentração $70 \%$.

Para os ensaios de resistência ao risco o polímero hidrofílico utilizado para recobrimento das amostras de PA11 foi o Hypol JM, da empresa DOW Chemical Company (EUA).

\section{Métodos}

\section{Tratamento dos tubos com chama}

O parâmetro variável no tratamento das amostras foi o tempo de exposição ao calor da chama. Os intervalos de tempo foram de $5,10,20,30$ e 60 segundos, valores dentro de uma faixa de tempo considerada adequada ${ }^{[13]}$.

$\mathrm{O}$ aparato utilizado no tratamento destes tubos de PA11 constituía-se de um rotor, uma haste para fixação das amostras e um maçarico. A Figura 1 apresenta um esquema simplificado do equipamento utilizado.

O procedimento de ensaio consistia em encaixar uma amostra de PA11 sobre a haste de fixação e, em seguida, ligar conjuntamente o maçarico e o rotor. As amostras giravam próximas à chama do maçarico pelo tempo predeterminado. A frequência de giro do rotor era de aproximadamente 73 RPM. O gás combustível utilizado no maçarico foi o butano. Apenas a região dos tubos de PA11 que sofreu tratamento por chama foi analisada.

\section{Caracterização por goniometria}

Para a análise da variação de energia livre de superfície das amostras, mensurada de forma indireta através do ângulo de contato, foi empregada a técnica de goniometria, realizada no Laboratório

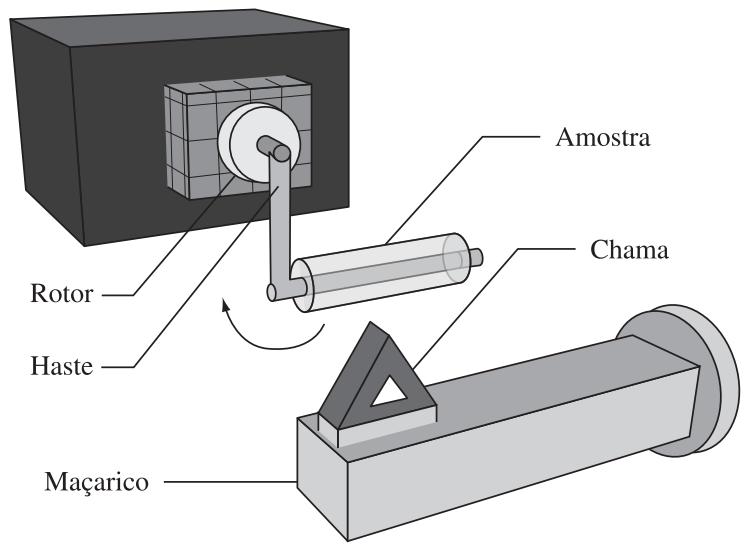

Figura 1. Equipamento utilizado para o tratamento de superfície com chama das amostras de tubos de PA11.

de Meios Porosos e Propriedades Termofísicas (LMPT) da UFSC. O equipamento utilizado foi um goniômetro Data Physics, modelo OCA-15, operado em condições ambientes de temperatura e pressão.

Sobre a superfície de cada amostra, tratada e não tratada, foram depositadas duas gotas de água deionizada (com volume de 5,0 $\mu \mathrm{L}$ cada) em regiões distintas, abrangendo uma área satisfatória para a avaliação. Após o tempo de 30 segundos para estabilização da gota, foram medidos dois ângulos por gota, totalizando quatro medidas por amostra. A imagem da gota foi capturada pela câmera de alta resolução do aparelho e um computador conectado ao sistema calculava automaticamente o valor desses ângulos através do software Image Tool.

\section{Análise por microscopia eletrônica de varredura}

Para a caracterização do relevo das amostras de PA11, bem como para a análise da resistência ao risco das amostras revestidas com Hypol JM, a técnica utilizada foi a microscopia eletrônica de varredura,realizadanoLaboratóriodeCaracterizaçãoMicroestrutural e Análise de Imagens (LCMAI) da UFSC. O aparelho utilizado foi o Microscópio Eletrônico de Varredura XL-30 da Philips.

Para a realização dos ensaios as amostras foram recobertas, antes das análises, com $20 \mathrm{~nm}$ de ouro no metalizador Bal-Tec modelo CED 030.

Os aumentos realizados foram de 50, 500, 3000 e 5000 vezes, para cada tempo estabelecido.

\section{Caracterização por Espectroscopia de Infravermelho com Transformada de Fourier por Reflectância (FTIR)}

Para se avaliar a composição química da superfície das amostras de PA11, antes e após o tratamento, a técnica empregada foi a de FTIR. A caracterização foi realizada no espectrômetro de infravermelho modelo $16 \mathrm{PC}$ da Perkin Elmer, pertencente à Central de Análises do Departamento de Química da UFSC. Os espectros gerados para identificação das ligações covalentes presentes no material foram obtidos a partir de varreduras sobre as amostras na região de 4000 até $400 \mathrm{~cm}^{-1}$, com penetração da onda eletromagnética de $2 \mu \mathrm{m}^{[2]}$.

\section{Recobrimento das amostras com polímero hidrofilico}

Para a aplicação do polímero hidrofílico sobre os tubos de PA11 foi construído um dispositivo composto de um aplicador de resina, um rotor, uma haste para fixação das amostras e uma lâmina para a regulagem da espessura da camada depositada. A Figura 2 apresenta um esquema simplificado do equipamento utilizado. 
Nesse procedimento, a amostra era fixada na haste, conectada ao eixo do rotor, e a lâmina ajustada a uma distância de $0,1 \mathrm{~mm}$ em relação a superfície da amostra. A amostra era movimentada a uma velocidade angular constante de 4,3 rad/s em relação à lâmina de ajuste. Sob estas condições o aplicador de resina liberava Hypol JM na superfície dos tubos até que os mesmos estivessem totalmente recobertos. Em seguida, os tubos eram mantidos girando no aparelho durante 1 hora para que ocorresse a cura do polímero hidrofílico.

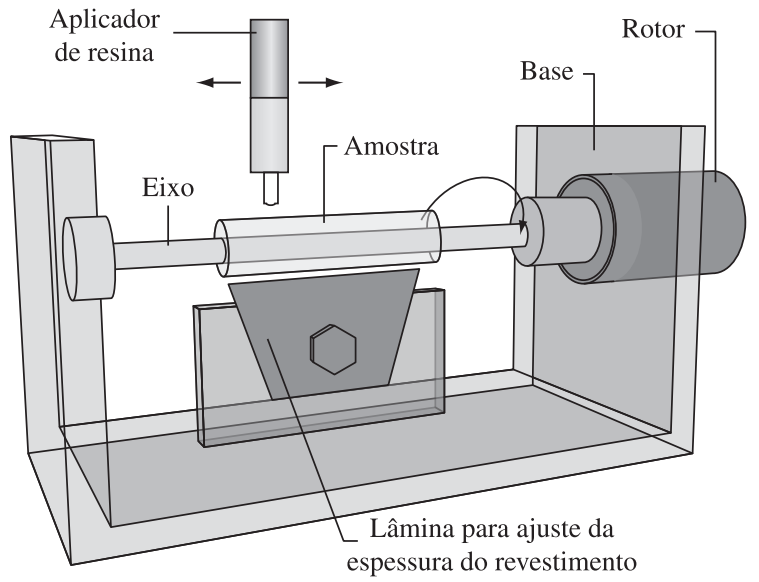

Figura 2. Dispositivo utilizado para a aplicação da camada hidrofílica sobre as amostras.

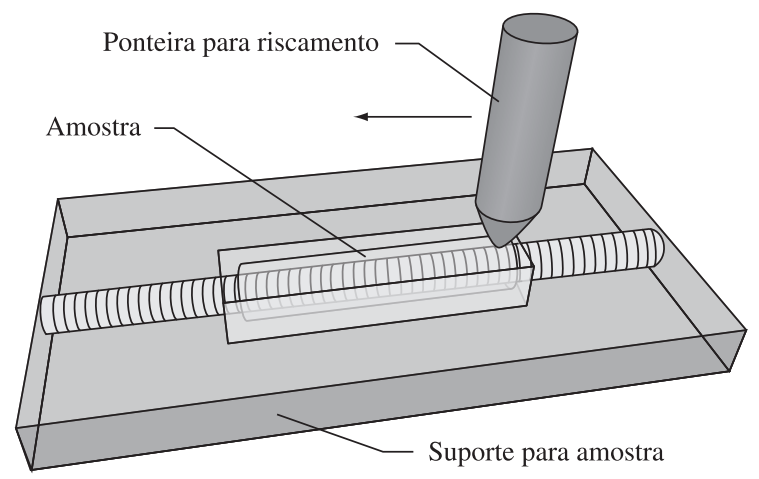

Figura 3. Esquema do aparelho empregado no teste de riscamento das amostras.

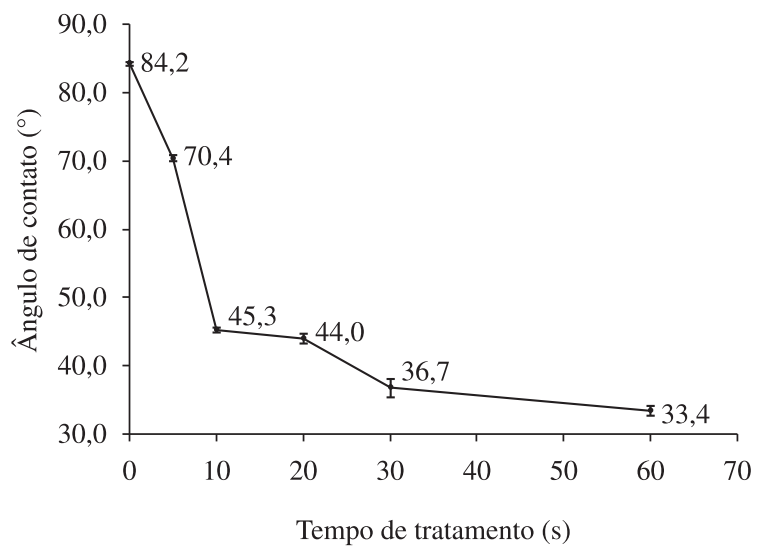

Figura 4. Ângulos de contato em função dos tempos de exposição ao tratamento com chama.

\section{Resistência ao risco}

Os ensaios de resistência ao risco foram realizados para que se pudesse observar o impacto direto do aumento da energia livre de superfície das amostras tratadas na adesão do polímero hidrofílico Hypol JM. Para este teste foi utilizada a amostra que apresentou o menor ângulo na análise goniométrica (60 segundos), bem como uma amostra não tratada. $\mathrm{O}$ aparato utilizado foi desenvolvido baseado na norma ASTM D 7027 ${ }^{[14]}$. A Figura 3 esquematiza este aparato.

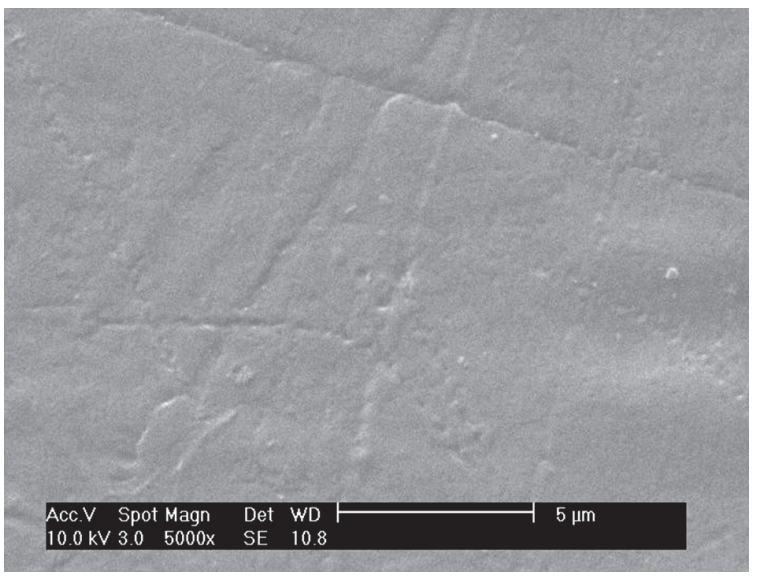

(a)

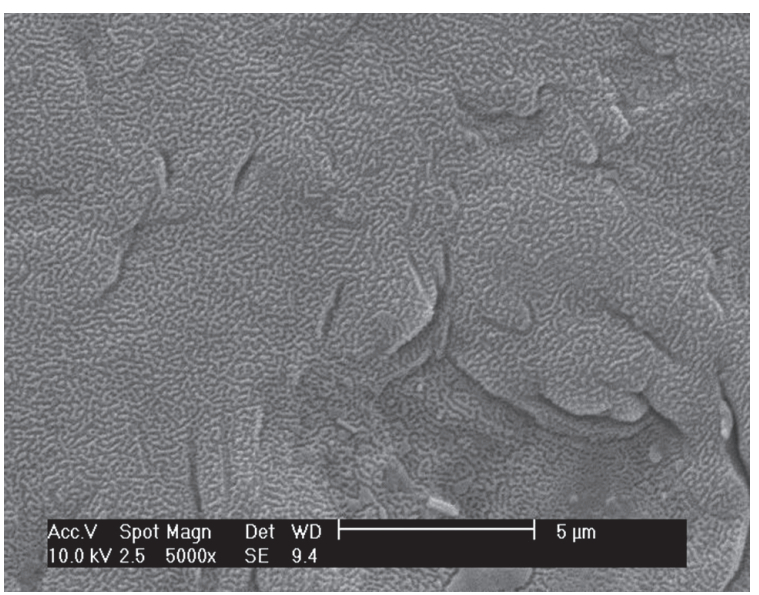

(b)

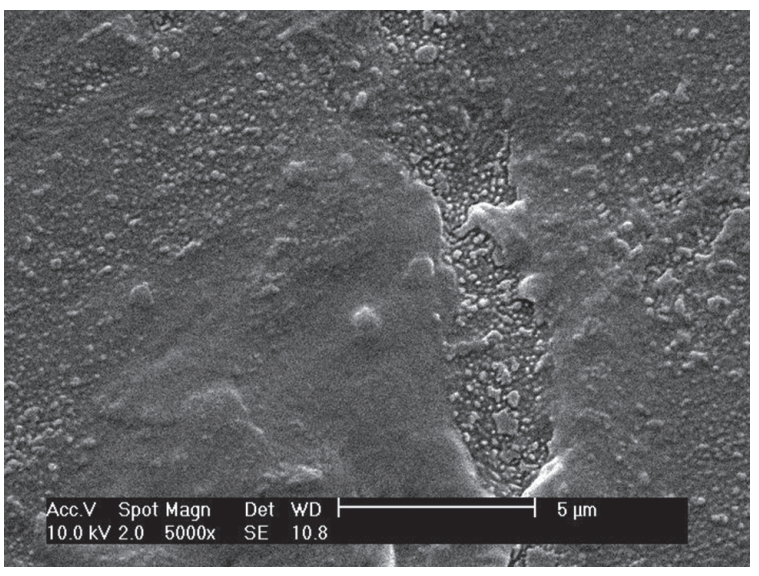

(c)

Figura 5. Micrografias das superfícies das amostras de PA11: a) não tratada; b) tratada durante 30 segundos; c) tratada durante 60 segundos. 
Os ensaios consistiam em fixar as amostras em um suporte e movimentar uma ponteira cônica metálica sobre a superfície dos tubos revestidos a uma velocidade constante. A ponteira era ajustada de tal forma que a profundidade dos riscos era equivalente a espessura da camada de polímero hidrofílico depositada sobre os tubos $(0,1 \mathrm{~mm})$. A análise dos resultados (riscos gerados na superfície das amostras) foi realizada de forma qualitativa através de imagens de microscopia eletrônica de varredura.

\section{Resultados e Discussão}

O gráfico da Figura 4 apresenta os resultados obtidos na análise goniométrica. Os ângulos representados na curva correspondem à média aritmética dos quatro valores de ângulo de contato obtidos para cada tempo. O tempo zero representa a amostra não tratada $\left(84,2^{\circ}\right)$. Como pode ser notado, houve uma diminuição gradativa dos valores dos ângulos de contato, de $84,2^{\circ}$ para $33,4^{\circ}$ (60 segundos de tratamento), à medida que o tempo de tratamento aumentou, com uma queda mais acentuada durante os primeiros 10 segundos. Um dos fatores que justifica essa diminuição dos ângulos de contato é o aumento da energia livre de superfície dos tubos após o tratamento, que provoca o aumento da interação eletrostática da superfície com as gotas depositadas, fazendo-as se espalharem (molhabilidade).

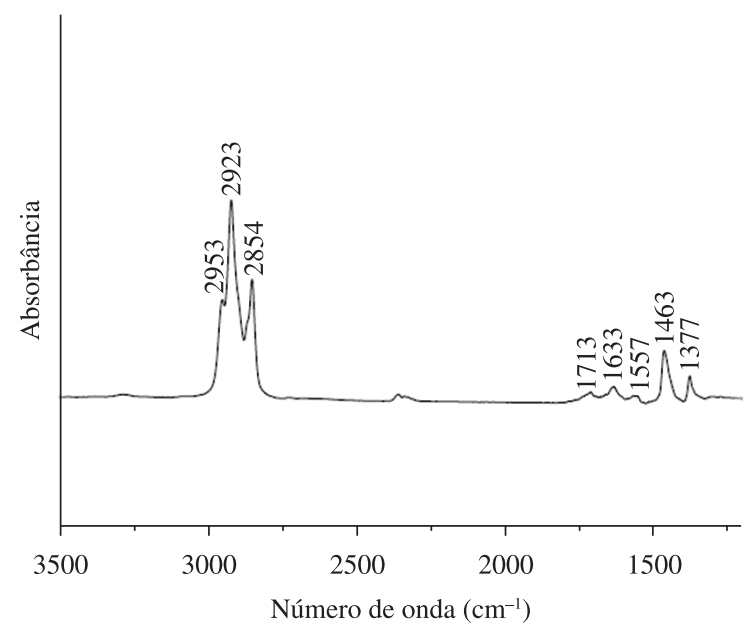

(a)

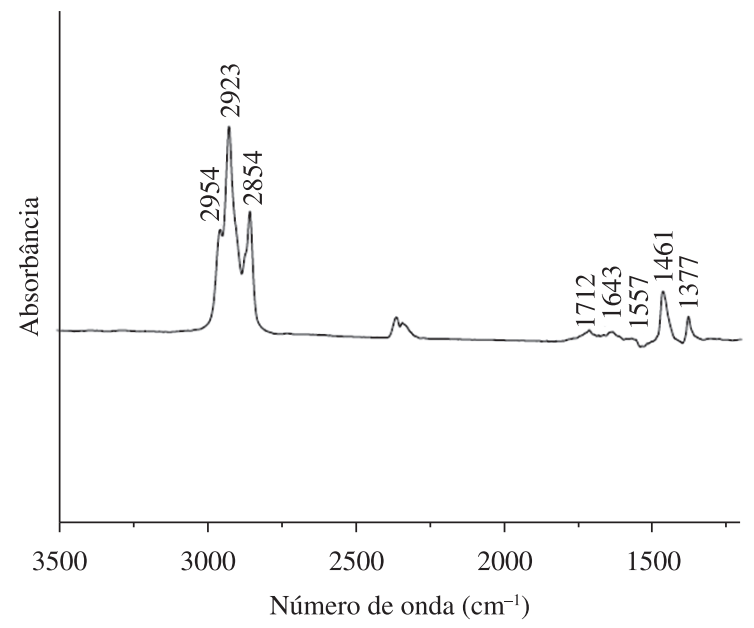

(b)

Figura 6. Espectros de infravermelho das amostras: a) não tratada; b) tratada durante 60 segundos.
A Figura 5 apresenta as micrografias obtidas por MEV da superfície da amostra tratada e não tratada, na ampliação de 5000×, a qual permitiu a melhor caracterização. Na Figura 5a, a amostra não tratada apresenta uma superfície plana, com apenas alguns leves riscos, provocados pelo manuseio do material. Nas micrografias (Figura 5b), correspondentes à amostra exposta durante 30 segundos, é possível notar mudanças significativas na textura das superfícies com o surgimento de rugosidades. Já na imagem (Figura 5c) (correspondente à amostra tratada durante 60 segundos) percebe-se além da presença de rugosidades, a formação de micro saliências arredondadas ao longo de sua superfície. As modificações na textura da superfície contribuíram para o aumento da molhabilidade (confirmada anteriormente pela análise goniométrica), pois ampliam a área de contato dos tubos, e consequentemente, aumenta a energia livre de superfície ${ }^{[1]}$.

Na Figura 6 observam-se os espectros de infravermelho gerados pela técnica FTIR da amostra não tratada (Figura 6a), e da amostra tratada por 60 segundos (Figura 6b), tempo que apresentou as modificações mais significativas tanto na diminuição do ângulo de contato quanto no aumento rugosidade. Os picos nas bandas intensas de absorbância correspondentes aos grupos funcionais presentes na estrutura molecular da PA11 estão entre 3500 e $1200 \mathrm{~cm}^{-1[15]}$.

Comparando-se ambos os espectros, é possível notar que ocorreram modificações na frequência de vibração de algumas ligações químicas após o tratamento, como por exemplo, de $2953 \mathrm{~cm}^{-1}$, correspondente à deformação axial assimétrica das

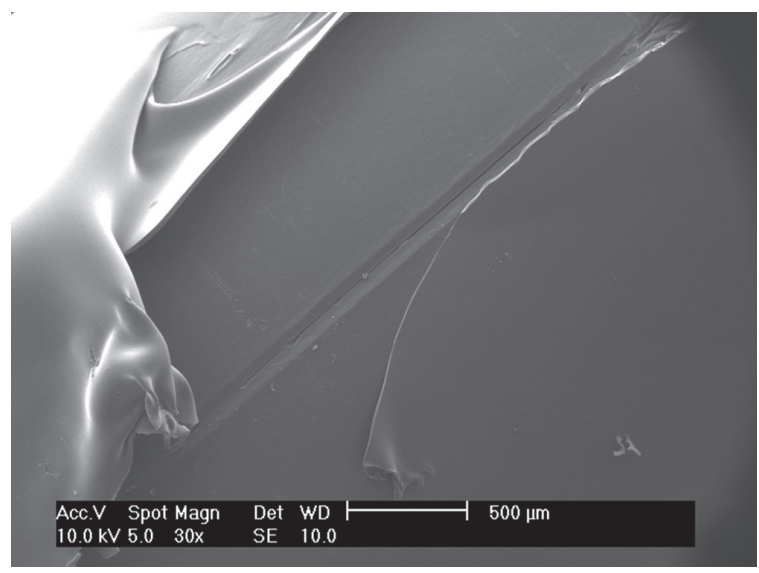

(a)

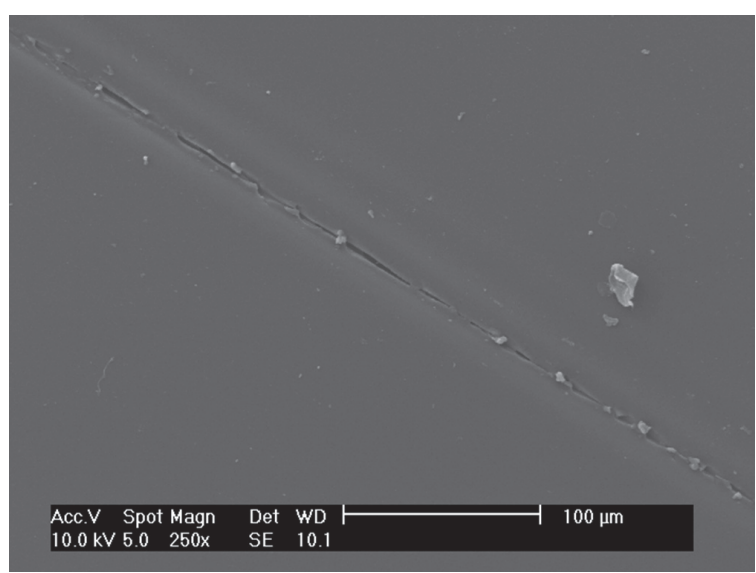

(b)

Figura 7. Micrografias das análises de risco das amostras: a) não tratada; b) tratada por chama durante 60 segundos. 
ligações $\mathrm{C}-\mathrm{H}$ do grupo $\mathrm{CH}_{3}$, para $2954 \mathrm{~cm}^{-1}$; de $1713 \mathrm{~cm}^{-1}$, banda referente à vibração de deformação axial de $\mathrm{C}=\mathrm{O}$, para $1712 \mathrm{~cm}^{-1}$; de $1633 \mathrm{~cm}^{-1}$, correspondente à vibração de deformação axial de $\mathrm{C}=\mathrm{O}$ (banda de amida $\mathrm{I}$ ) para $1631 \mathrm{~cm}^{-1}$ e de $1557 \mathrm{~cm}^{-1}$, referente à vibração de deformação angular do $\mathrm{N}-\mathrm{H}$ (banda de amida II), para $1567 \mathrm{~cm}^{-1}$. Observa-se ainda que em outros casos as bandas não sofreram modificações, como é o caso da banda em $2923 \mathrm{~cm}^{-1}$ correspondem à deformação axial assimétrica das ligações $\mathrm{C}$-H do grupo $\mathrm{CH}_{2}$, em $2854 \mathrm{~cm}^{-1}$, referente à deformação axial simétrica de ligações $\mathrm{C}-\mathrm{H}$ do grupo $\mathrm{CH}_{2}$, em $1463 \mathrm{~cm}^{-1}$ refere-se à deformação angular simétrica do $\mathrm{CH}_{2}$, e em $1377 \mathrm{~cm}^{-1}$ correspondente à deformação angular simétrica do $\mathrm{CH}_{3}$. Não é possível evidenciar, a partir destas pequenas alterações em alguns números de onda, que ocorreram modificações significativas na estrutura molecular das amostras de PA11 após o tratamento.

As micrografias da Figura 7 mostram a superfície da amostra não tratada (Figura 7a) e tratada durante 60 segundos (Figura 7b) que foram recobertas com a camada hidrofílica e submetidas ao teste do riscamento. Como pode ser observado na Figura 7a, nota-se um desprendimento da camada hidrofílica em praticamente toda a extensão do risco, comprovando a baixa aderência entre esses dois materiais. Já na imagem do risco feito sobre a amostra tratada, notase que este mesmo riscamento provocou apenas a abertura de um sulco bem discreto na camada hidrofílica, comprovando assim a melhora significativa na aderência do revestimento hidrofílico após o tratamento.

\section{Conclusões}

Nas condições apresentadas neste estudo, o tratamento de superfície de tubos de poliamida 11 através do processo de exposição à chama mostrou-se adequado na preparação da superfície desse material para receber a deposição de uma camada polimérica hidrofílica biomédica (Hypol JM), uma vez que esta técnica gerou um aumento significativo na energia livre de superfície do material (medido de forma indireta pela diminuição do ângulo de contato). A partir dos espectros de infravermelho obtidos na análise de FTIR após o tratamento não é possível afirmar que ocorreram modificações significativas na estrutura molecular das amostras de PA11, porém, os mecanismos de alterações de superfície (aumento da rugosidade (imagens de MEV) e a diminuição dos ângulos de contato (análise goniométrica) confirmaram o aumento da energia livre de superfície da PA11. Estas duas últimas análises permitiram constatar ainda que as alterações de superfície foram diretamente dependentes do tempo de exposição à chama, sendo o tratamento durante 60 segundos aquele que apresentou o melhor resultado (maior grau de rugosidade e maior diminuição do ângulo de contato, $\left.33,4^{\circ}\right)$.

\section{Agradecimentos}

Os autores agradecem à empresa Nano Endoluminal S.A., patrocinadora do projeto, ao Laboratório de Projeto e Fabricação de Componentes de Plástico Injetados (CIMJECT) da UFSC, ao Professor Dr. Celso Peres Fernandes e ao Laboratório de Meios Porosos e Propriedades Termofísicas (LMPT) da USFC.

\section{Referências Bibliográficas}

1. Laporte, R. J. - "Hydrophilic polymer coatings for medical devices: structure/properties, development, manufacture and applications", CRC PRESS, New York (1997).

2. Baijal, M. D. - "Plastics Polymers Science and Technology", John Wiley \& Sons, New York (1982).

3. Öztürk, G. I. - "Selected Methods of Surface Engineering Applied to Materials Science", Dissertação de Mestrado, Sabancı University, Turquia (2004).

4. Burakowski, L. \& Rezende, M.C. - Polímeros, 11, 2, p.51-57 (2001).

5. Sheng, E.; Sutherland, I.; Brewis, D. M.; Heath, R. J. \& Bradley, R. H. - Journal of Materials Chemistry, 3, p.487-490 (1994).

6. Kilic, B.; Aksit, A.C. \& Mutlu, M. International Journal of Clothing Science and Technology, 21, p.137-145 (2009).

7. Desmet, T.; Morent, R.; De Geyter, N.; Leys, C.; Schacht, E. \& Dubruel, P. -Biomacromolecules, 10, 9, p.2351-2378 (2009).

8. Hao, L. \& Lawrence, J. - "Laser Surface Treatment of Bio-Implant Materials”, Wiley, New Jersey (2005).

9. Waugh, D. G.; Lawrence, J.; Walton, C. D. \& Zakaria, R.B. - Optics \& Laser Technology, 42, p. 347-356 (2010).

10. Acquarulo, L. A. Jr.; O’Neil, C. J. \& Nilajkar, A. S. - "Lubricious compounds for biomedical applications using hydrophilic polymers", World Intellectual Property Organization, Switzerland (2006).

11. Van Krevelen, D. W. - "Properties of polymers: their correlation with chemical structure; their numerical estimation and prediction from additive group contributions", Elsevier Science, Amsterdam (1997).

12. Dorai, R. \& Kushner, M. J. - Journal of Physics D: Applied Physics, 36, p. 666-685 (2003).

13. Gutowski, W. S.; Wu, Dong Y. \& Li, S. - "Surface Treatment of Polymers", Patent Number: 5.879.757, United States Patent (1999).

14. ASTM D7027 - "Standard Test Method for Evaluation of Scratch Resistance of Polymeric Coatings and Plastics Using an Instrumented Scratch Machine", West Conshohocken (2005).

15. Silverstein, R. M.; Bassler, G. C. \& Morrill, T. C. - "Spectrometric Identification of Organic Compounds", John Wiley \& Sons, New York (1997).

Enviado: 09/09/09

Reenviado: $19 / 02 / 10$

Aceito: $22 / 02 / 10$

DOI: $10.1590 / \mathrm{S} 0104-14282010005000030$ 